تحضير مرادف حيوي من خميرة Saccharomyces cerevisiae وإستخد(مه في خفض الإصابة التجريبية بجراثيم Salmonella typhimurium في أفراخ اللحم 2- عند عمر متقدم (16 - 30 يوم) ئهم)

غادة عبد الخالق قطان و فارس عبد علي العبيدي و شهرزاد محمد الثديدي وحدة الامراض المشتركة - كلية الطب البيطري - جامعة بغداد - بغداد - العراق

\title{
الخلاصة
}

استهذف البحث تحضير مرادف حيوي من خميرة Saccharomyces cerevisiae وتقييم استخدامه بنسبتي 0.1 , 0.2 \% من العلف (T2 و T3 ) لخفض الاصابة التجريبية لأفراخ اللحم

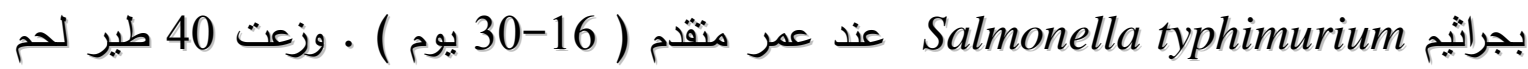

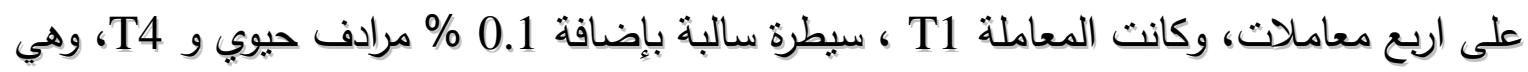

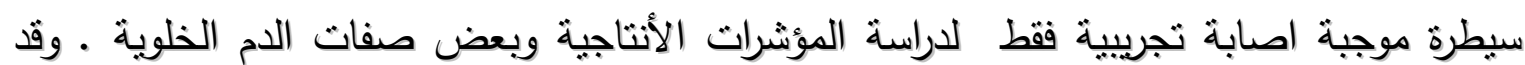

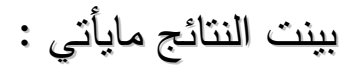

حضر مرادف حيوي لخميرة Sacch. cerevisiae يحوي الغرام الواحد على 10 حلية خميرة Sacch. cerevisiae 10 ملغم من متعدد السكر مانان / غم مرادف حيوي ـ أدى إستخدام 0.1 و 0.2 \% منه الى خفض شدة الإصابة التجريبية من خلادل نحسين وزن الجسم والزيادة الوزنية وكفاءة التحويل ، وخفض نسبة الهلاكات وخفض الأجهاد الفسلجي الناتج عن الإصابة|لتجريبية

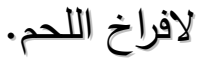




\section{Preparing of saccharomyces cereviciae Synbiotic for Reducing Expermental Infection of salmonella typhimurium in Broiler 2 - advanced age ( 16 -30 days)}

\section{Ghada A. Qatan , Faris A. Al-Obaidi and Shahrazad M. Al-Shadeedi} Zoonosis unit - College of Vet. Med.- Baghdad University - Baghdad - Iraq

\section{Summary}

This study aimed to prepare a synbiotic of Sacch. cerevisiae and used as 0.1 and $0.2 \%$ in feed (T2,T3) for reducing the experimental infection of Salmonella typhimurium to broiler chicks ( $16-30$ days) by using 40 birds divided into four treatments, T1 was negative control with $0.1 \%$ synbiotics and $\mathrm{T} 4$ was positive control with experimental infection only, production and blood parameters were studied, and the results showed the following:

A synbiotic of Sacch. cerevisiae was made which have $10^{7} \mathrm{cfu}$ of Sacch. cerevisiae and $10 \mathrm{mg}$ Mannan Oligosaccharide per gram .Using 0.1 and $0.2 \%$ of Sacch. cerevisiae synbiotic reduced experimental infection of Salmonella typhimurium via increase body weight and feed conversion and decrease mortality and physiological stress of experimental infection.

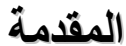

المرادف الحيوي (Synbiotic) منتوج خليط من المعزز الحيوي (Probiotic) والسابق الحيوي

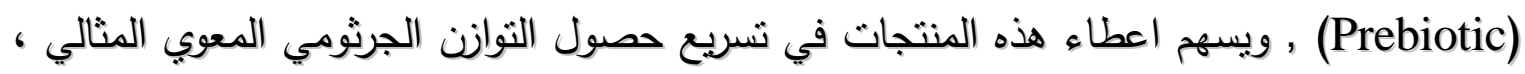

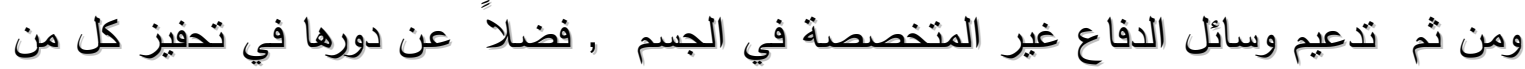

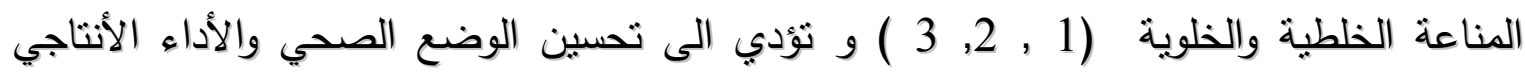

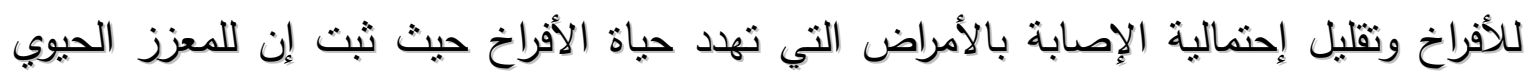
(Probiotic) القولون (6) ، وكذلك خفض الأصابة بالأمراض التي نسبيها جراثيم السالمونيلا ، (7 ) ، وهذه بدورها بالية

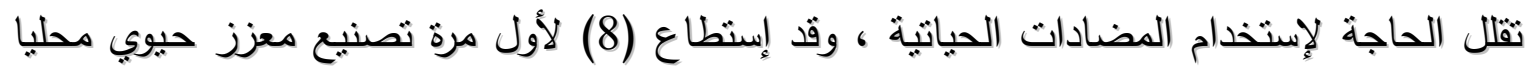

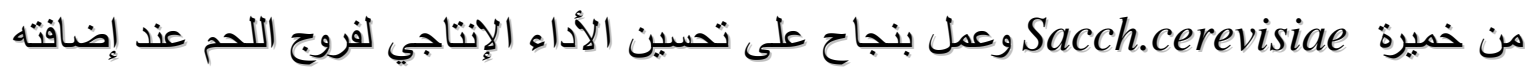
Sacch. بنسبة 2,1 كغم / طن علف ؛ لذا يهدف البحث الحالي تحضير مرادف حيوي من خميرة S. والسكر متعدد المانان وتقييم اثر اضافته في خفض الإصابة التجريبية بجراثيم cerevisiae 
typhimurium

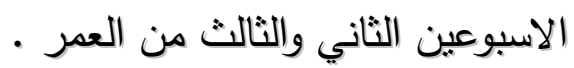

\section{المواد وطرائق العمل}

\section{Sacch.cerevisiae تحضير المرادف الحيوي لخميرة}

استخدمت خميرة Sacch. cerevisiae الجافة التجارية نوع Sakmaya نركية المنشأ لتحضير المعزز الحيوي حسب ماذكرت (8) , ثم اضيف له الجدار الخلوي لمستتبث الخميرة المنماة

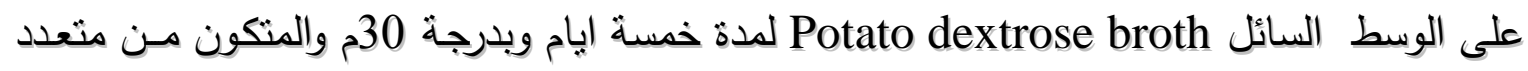
السكريات مانان (Mannan Oligosaccharids) كسابق حيوي للحصول بالنتيجة على مرادف

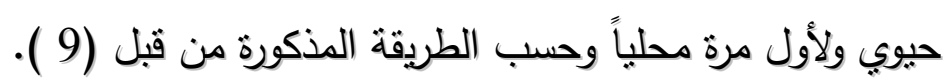

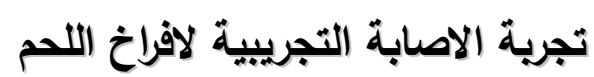
اجري البحث في بيت الحيوان في كليـة الطب البيطري خـلال المدة من 15 - 30 حزيـران

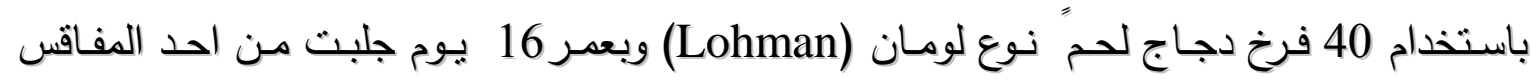

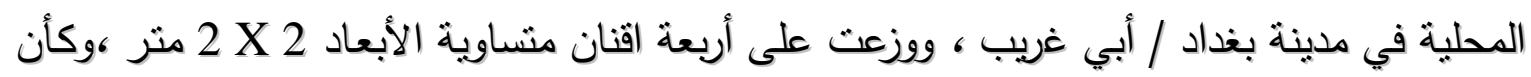

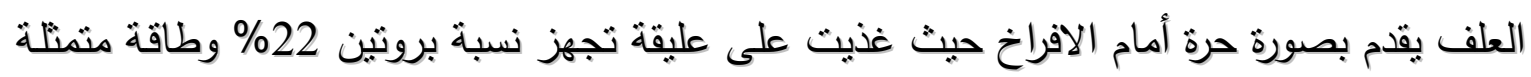
3044 كيلوسعرة لكل كيلوغرام علف ، وكانت المعاملات كالأتي :

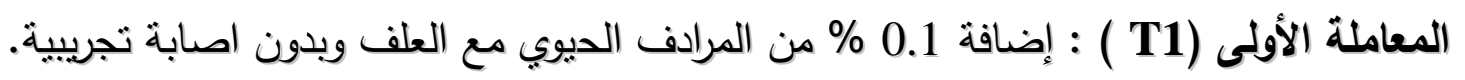

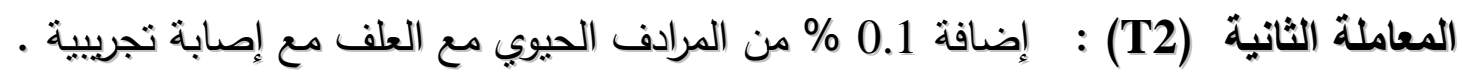

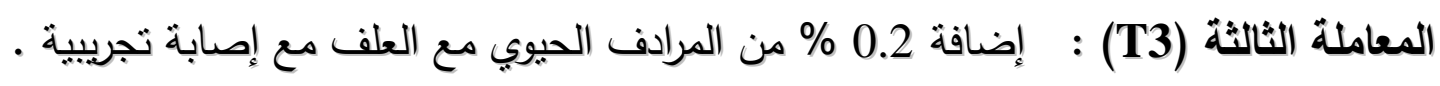
المعاملة الرابعة (T4) : إصابة تجريبية فقط . جراثيم جرعة التحدي:

استخدمت جراثيم S.typhimurium كان الحصول عليها عن طريق مختبر الصحة المركزي /

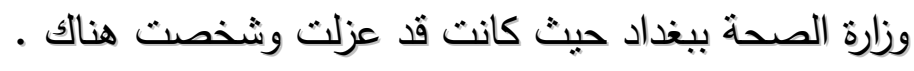
تحضير جرعة التحدي:

S. كضرت جرعة التحدي حسب طريقة (10) حيث أخذت 5 مستعمرات جرثومية نقية من

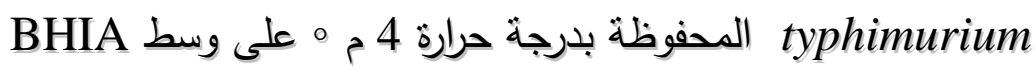
(Brain Heart Infusion Agar) (Nutrient Broth) NB نحوي على 5 مل من المرق المغذيت (Universal Bottle) المحتويات بثكل جيد وحضنت بدرجة حرارة 37م لمدة 24 ساعة ثم مزجت محتويات القناني 
الخمس في دورق معقم ،وأخذ 0.1 مل منها وزرع في قنينة قياسية معقمة تحوي 9 مل من المرق

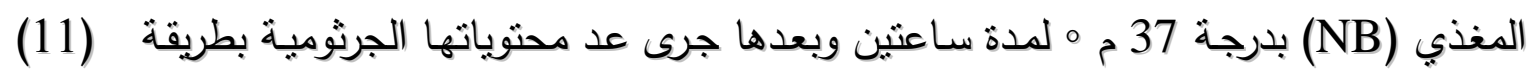

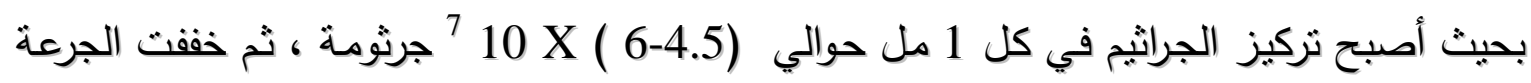

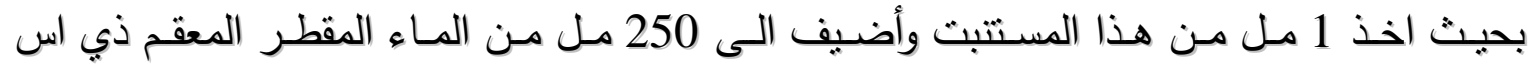

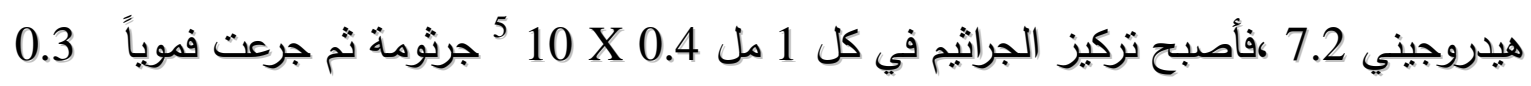

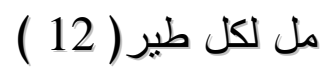
الصفات المدروسة مل لايرة الصفات الأنتاجية لأفراخ اللحم المدرون وزنت الأفراخ بصورة فردية جميعاً في كل مكرر منذ اليوم الأول للتجربة وعند نهاية كل أسبوع

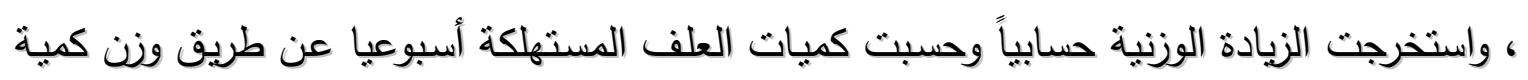

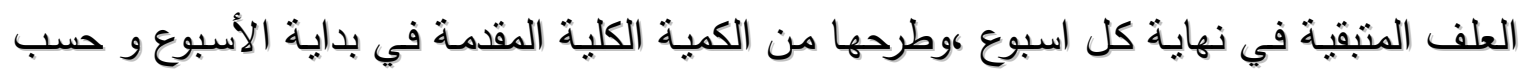

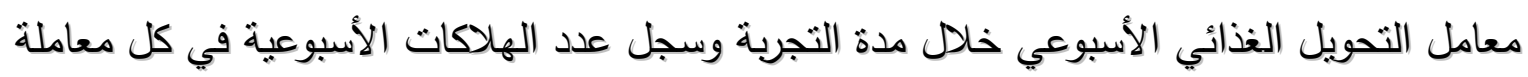
وحسبت نسبتها المئوية . فحوصات الام الخلوية

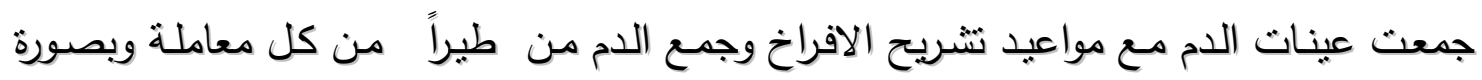

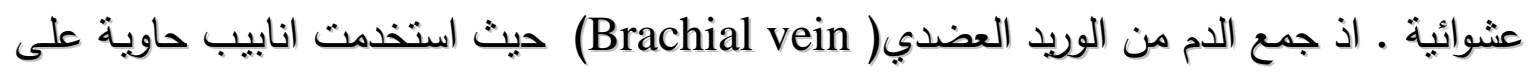

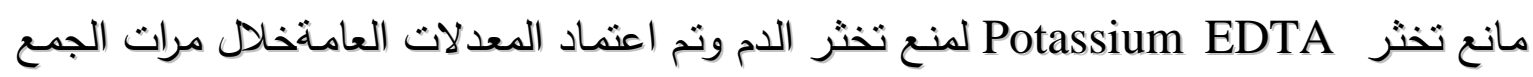

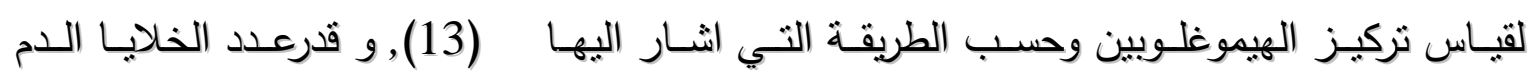

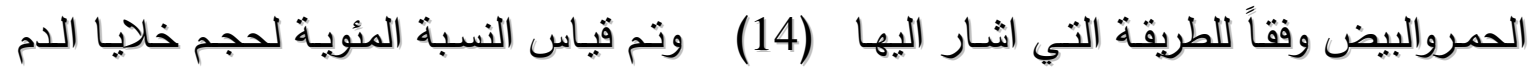
المرصوصة حسب الطريقة الني اثشار اليها (15) . التحليل الأحصائي :

حلات البيانات وفق التصميم العشوائي الكامل (Complete Randomized Design)، وجرى مقارنة المتوسطات وفق أختبار دنكن متعدد المديات • وبإِتخدام التحليل الأحصائي الجاهز (16) SAS (Duncan's multiple range test) 
النتائج

تحضير المرادف الحيوي

تم وبنجاح تحضير اول مرادف حيوي محلياً من خميرة Sacch. cerevisiae يحوي على الى

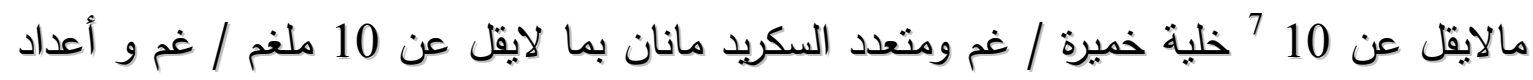

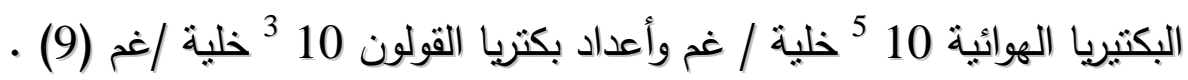
تطبيق استخدام المرادف الحيوي في خفض الاصابة التجريبية بجراثيم S.typhimurium في

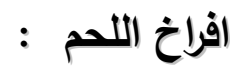

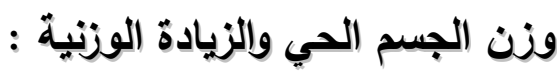
من الجدول (جدول1) ان المعاملة T1 قد سجلت اعلى وزن جسم عند عمر ثلاثة واربعة اسابيع، وكانت 350 و 530 غم على التوالي وبفارق معنوي (P<0.05) مقارنة بافراخ المعاملة

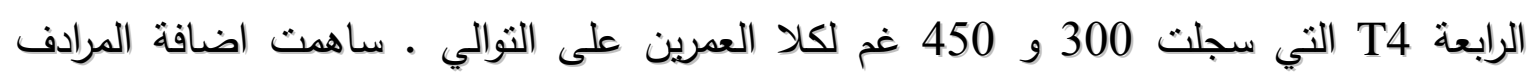

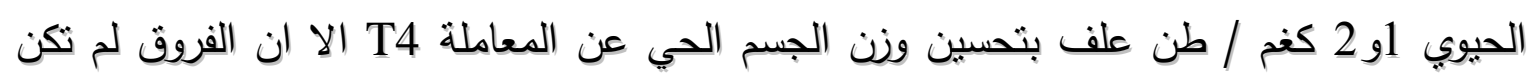

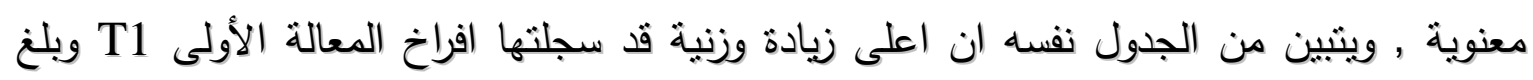

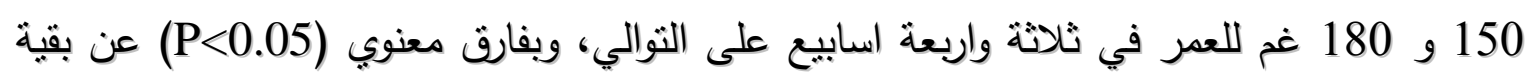

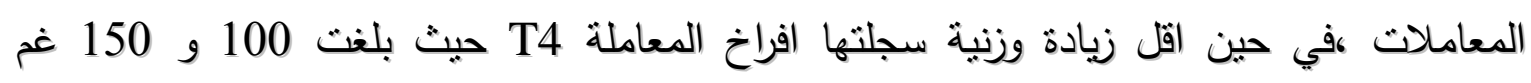

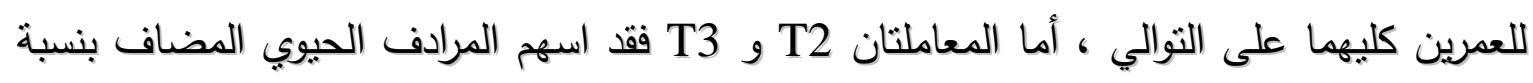

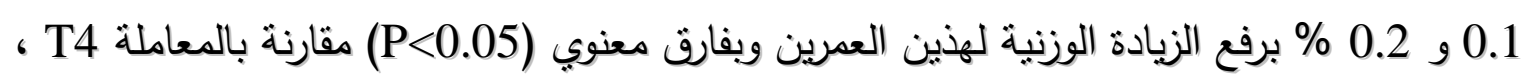

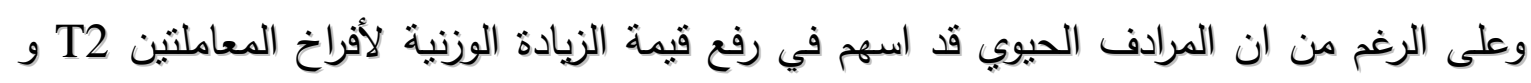

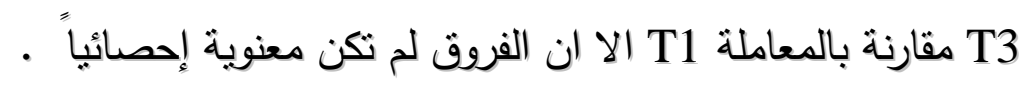


جدول(1) :معدل الوزن الحي والزيادة الوزنية لأفراخ اللحم بعد الإصابــة التجريبيـة بجراثيم 30-16 بوم S. typhimurium

\begin{tabular}{|c|c|c|c|c|}
\hline 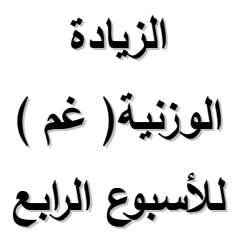 & 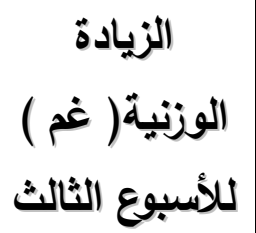 & معدل الوزن الحي & معدل الوزن الحي & المعاملات \\
\hline $\begin{array}{c}180 \\
\pm 1.36 \\
\mathrm{a}\end{array}$ & $\begin{array}{c}150 \\
\pm 1.22 \\
\quad \mathrm{a}\end{array}$ & $\begin{array}{c}530 \\
\pm 10.24 \\
\mathrm{a}\end{array}$ & $\begin{array}{c}350 \\
\pm 5.14 \\
\text { a }\end{array}$ & T1 (Cont.) \\
\hline $\begin{array}{c}170 \\
\pm 1.26 \\
\mathrm{~b}\end{array}$ & $\begin{array}{c}130 \\
\pm 1.00 \\
\mathrm{~b}\end{array}$ & $\begin{array}{c}500 \\
\pm 10.17 \\
\mathrm{ab}\end{array}$ & $\begin{array}{c}330 \\
\pm 5.09 \\
\mathrm{ab}\end{array}$ & 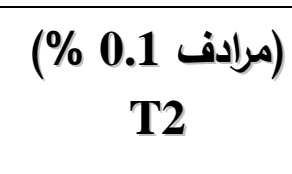 \\
\hline $\begin{array}{c}174 \\
\pm 1.33 \\
\mathrm{~b}\end{array}$ & $\begin{array}{c}136 \\
\pm 1.25 \\
\mathrm{~b}\end{array}$ & $\begin{array}{c}510 \\
\pm 11.25 \\
\mathrm{ab}\end{array}$ & $\begin{array}{c}330 \\
\pm 5.11 \\
\mathrm{ab}\end{array}$ & (مرادف 0.2 \% \\
\hline $\begin{array}{c}150 \\
\pm 1.26 \\
\mathrm{c}\end{array}$ & $\begin{array}{c}100 \\
\pm 1.09 \\
\mathrm{c}\end{array}$ & $\begin{array}{c}450 \\
\pm 11.29 \\
\mathrm{~b}\end{array}$ & $\begin{array}{c}300 \\
\pm 5.10 \\
\mathrm{~b}\end{array}$ & (إصابة فقط () \\
\hline$*$ & $*$ & $*$ & $*$ & المعنوية \\
\hline
\end{tabular}

الأحرف الصغيرة المختلفة ضمن كل عمود تثثير الى وجود فرق معنوي .

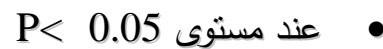

أستهلاك العلف وكفاءة التحويل الغذائي :

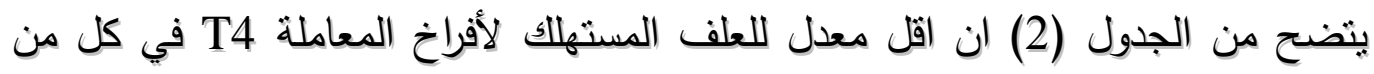
الأسبوعين الثالث والرابع من العمر حيث بلغ 280 و 455 غم / طير / اسبوع على النوالي وبفارق

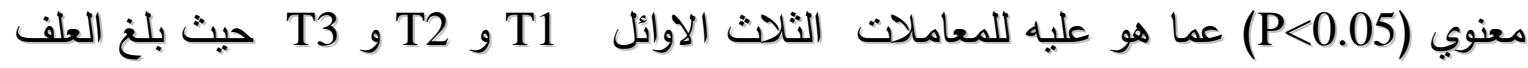

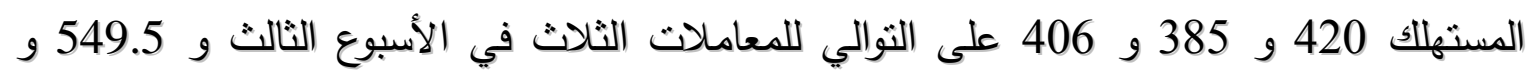

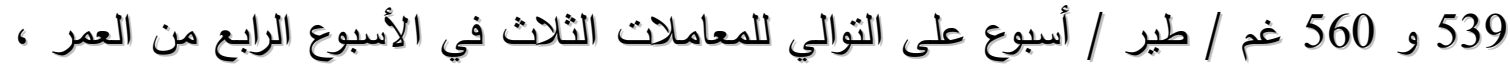

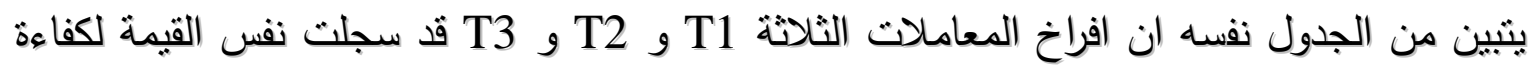

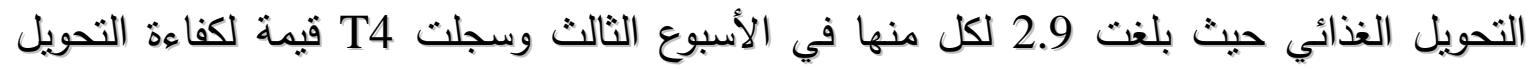

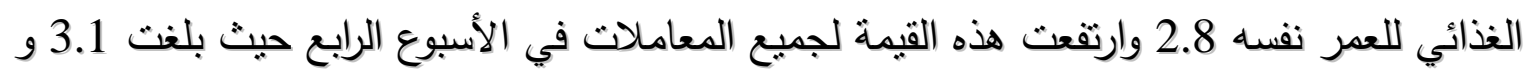


3.1 و 3.2 و 3.0 للمعاملات T1 و T2 و T3 و T4 على التوالي ولم يكن هنالك فروق معنوية

إحصائيا .

جدول (2): استهلاك العلف وكفاءة التحويل الغذائي لإفراخ اللحم بعد الإصابة التجريبية بجراثيم 30-16 بعمر S. typhimurium

\begin{tabular}{|c|c|c|c|c|}
\hline كفاعة التحويل & كفاءة التحويل & 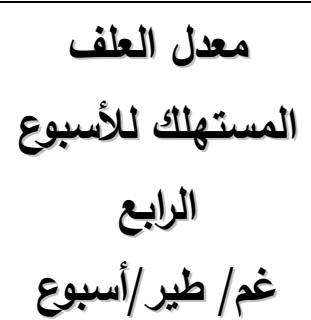 & 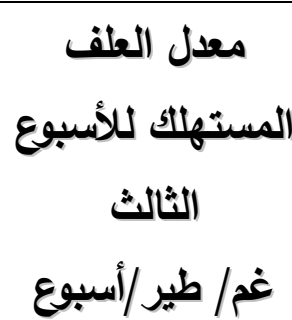 & المعاملات \\
\hline $\begin{array}{c}3.1 \\
\pm 0.21 \\
\mathrm{a}\end{array}$ & $\begin{array}{c}2.9 \\
\pm 0.25 \\
a\end{array}$ & $\begin{array}{c}549.5 \\
\pm 9.33 \\
\mathrm{a}\end{array}$ & $\begin{array}{c}420 \\
\pm 3.95 \\
\mathrm{a}\end{array}$ & T1 (Cont.) \\
\hline $\begin{array}{c}3.1 \\
\pm 0.20 \\
\quad a\end{array}$ & $\begin{array}{c}2.9 \\
\pm 0.31 \\
\quad \mathrm{a}\end{array}$ & $\begin{array}{c}539 \\
\pm 9.25 \\
\mathrm{~b}\end{array}$ & $\begin{array}{c}385 \\
\pm 3.85 \\
b\end{array}$ & $\begin{array}{c}\text { (مرادف } 0.1 \text { (م2) } \\
\text { T2 }\end{array}$ \\
\hline $\begin{array}{c}3.2 \\
\pm 0.20 \\
\quad \mathrm{a}\end{array}$ & $\begin{array}{c}2.9 \\
\pm 0.11 \\
\quad \mathrm{a}\end{array}$ & $\begin{array}{c}560 \\
\pm 8.61 \\
b\end{array}$ & $\begin{array}{c}406 \\
\pm 4.10 \\
\mathrm{~b}\end{array}$ & (مرادف 0.2 \% \\
\hline $\begin{array}{c}3.0 \\
\pm 0.20 \\
\quad \mathrm{a}\end{array}$ & $\begin{array}{c}2.8 \\
\pm 0.19 \\
\quad \mathrm{a}\end{array}$ & $\begin{array}{c}455 \\
\pm 8.91 \\
c\end{array}$ & $\begin{array}{c}280 \\
\pm 3.82 \\
\quad \mathrm{c}\end{array}$ & $\begin{array}{l}\text { (إصابة فقط (44 } \\
\text { T4 }\end{array}$ \\
\hline$*$ & $*$ & $*$ & $*$ & المعنوية \\
\hline
\end{tabular}

الأحرف الصغيرة المختلفة ضمن كل عمود تشير الى وجود فرق معنوي .

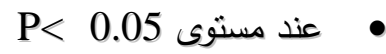

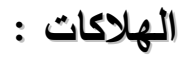

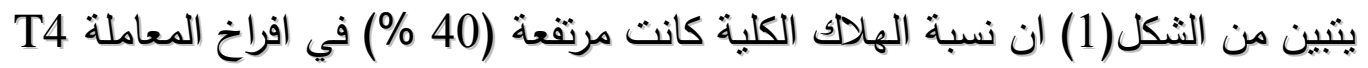
وبفارق عالي المعنوية (P<0.01) عن بقية المعاملات وفي الوقت نفسه سجلت المعاملة T1 أدنى نسبة هلاكات كلية إذ بلغت 12.5 \% , وقد اسهمت معاملات اضافة او 2 كغم من المرادف

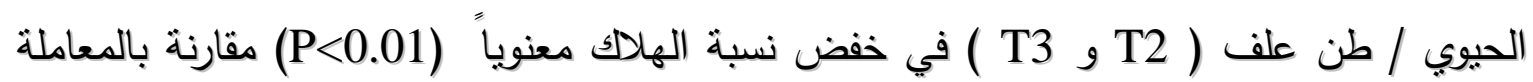
T4 اذ سجلتا 26 و 20 \% على التوالي . 
صفات الام الخلوية

يتضح من الجدول (3) إن اعلى عدد لخلايا الدم الحمر(RBCs) سجلته أفراخ المعاملة

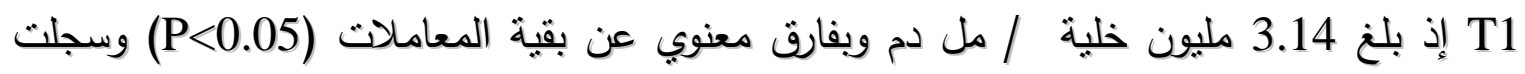
معاملتي T2 و T2 2.91 و 2.93 مليون خلية / مل دم على الثوالي في حين كانت ماسجلته

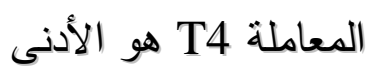

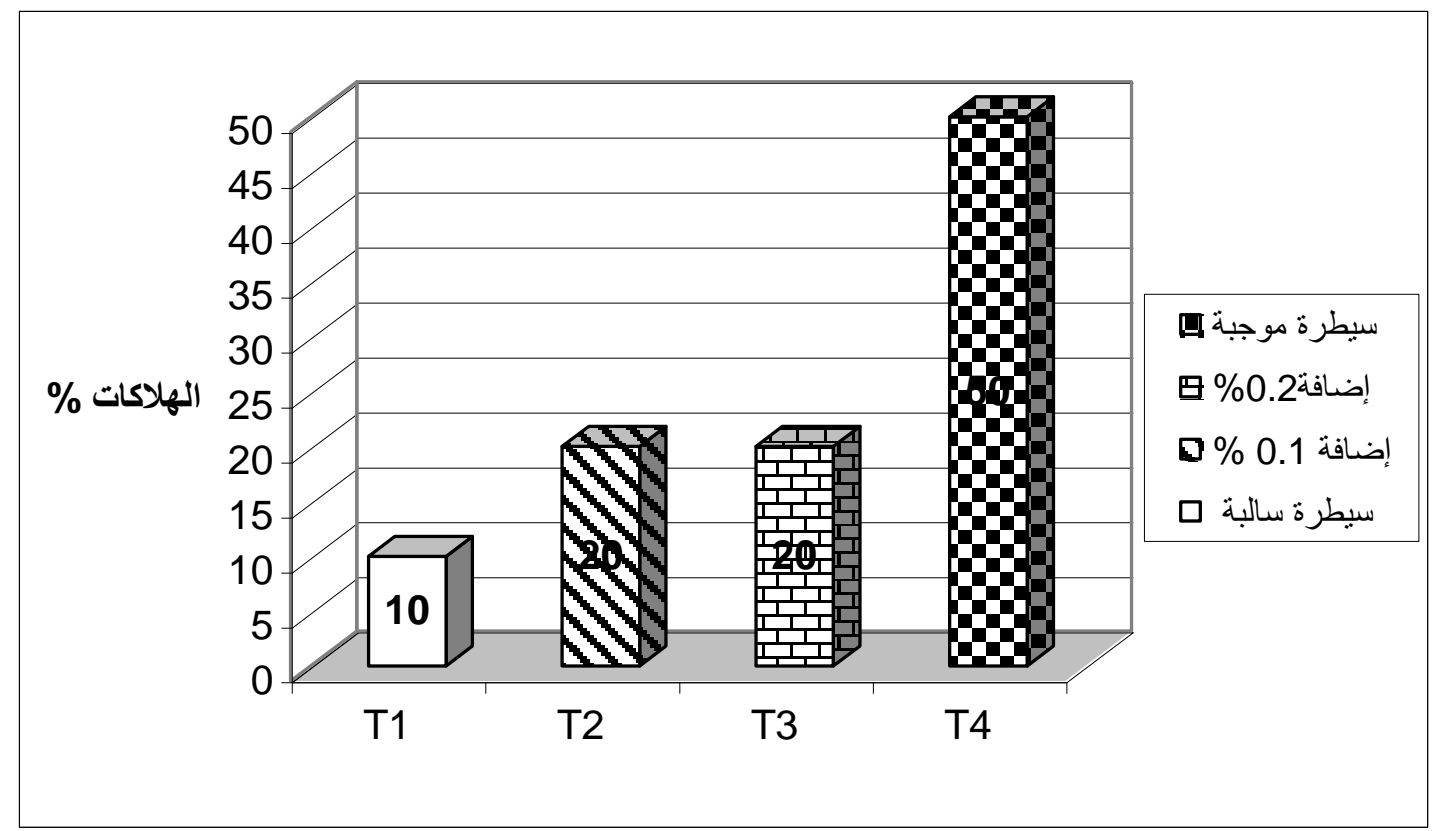

شكل (1) :يبين تأثير اضافة المرادف الحيوي في نسبة الهلاكات الكلية لأفراخ اللحم بعد الإصابة

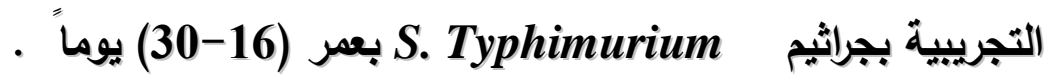

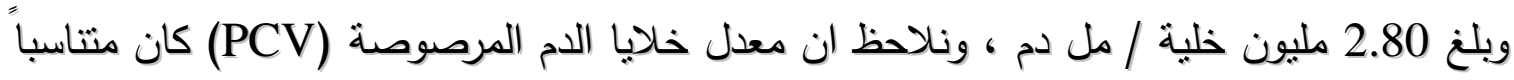

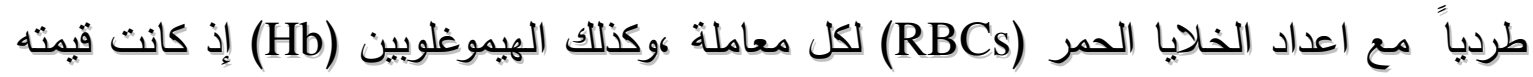
أكثر ارتفاعاً في افراخ المعاملة T1 ( 7.95 غم / 100 مل دم) وأكثر انخفاضاً لافراخ المعاملة T4

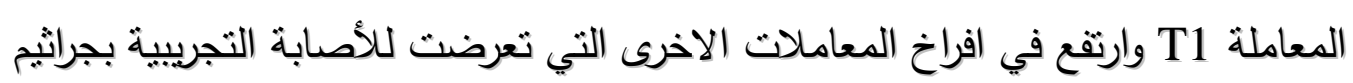
S. typhimurium اعداد هذه الخلايا ا 


\section{المناقشة}

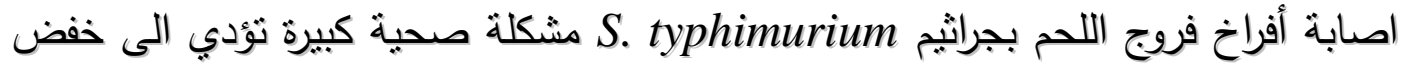

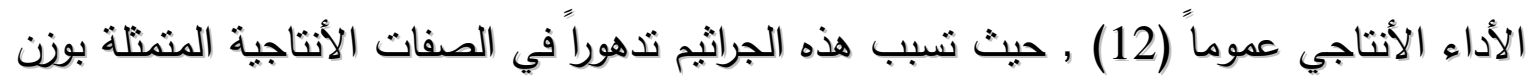

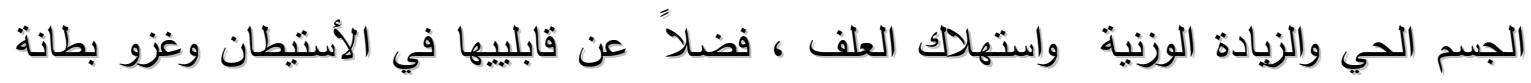

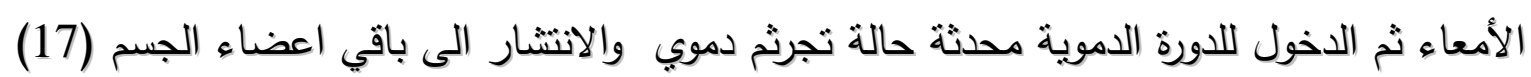

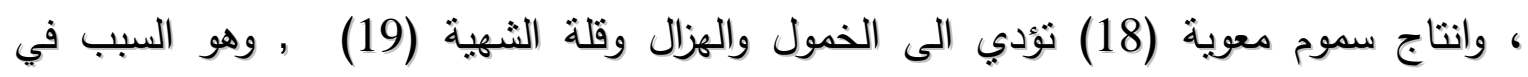

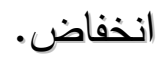

جدول (3): بعض صفات الدم الخلوية لأفراخ اللحم المصابة تجريبياً بجراثيم S typhimurium

\begin{tabular}{|c|c|c|c|c|}
\hline غم & خلية / مل دم الف & PCV \% & 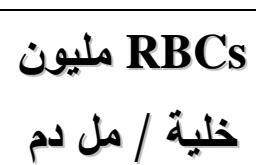 & المعاملات \\
\hline $\begin{array}{c}7.95 \\
\pm 0.30 \\
\quad \mathrm{a}\end{array}$ & $\begin{array}{c}23.17 \\
\pm 1.45 \\
\mathrm{c}\end{array}$ & $\begin{array}{c}35.0 \\
\pm 0.52 \\
\mathrm{a}\end{array}$ & $\begin{array}{c}3.14 \\
\pm 0.44 \\
\text { a }\end{array}$ & T1 (Cont.) \\
\hline $\begin{array}{c}7.68 \\
\pm 0.31 \\
b\end{array}$ & $\begin{array}{c}23.95 \\
\pm 1.45 \\
\mathrm{~b}\end{array}$ & $\begin{array}{c}34.3 \\
\pm 0.56 \\
\mathrm{~b}\end{array}$ & $\begin{array}{c}2.91 \\
\pm 0.39 \\
b\end{array}$ & $\begin{array}{c}\text { (مرادف } 0.1 \text { (م2) } \\
\text { T2 }\end{array}$ \\
\hline $\begin{array}{c}7.71 \\
\pm 0.31 \\
\mathrm{~b}\end{array}$ & $\begin{array}{c}23.96 \\
\pm 1.36 \\
\mathrm{~b}\end{array}$ & $\begin{array}{c}34.6 \\
\pm 0.66 \\
\mathrm{~b}\end{array}$ & $\begin{array}{c}2.93 \\
\pm 0.38 \\
\mathrm{~b}\end{array}$ & $\begin{array}{c}\text { \% } 0.2 \text { (مرادف) } \\
\text { T3( }\end{array}$ \\
\hline $\begin{array}{c}7.31 \\
\pm 0.29 \\
c\end{array}$ & $\begin{array}{c}24.25 \\
\pm 1.39 \\
\quad \mathrm{a}\end{array}$ & $\begin{array}{c}33.5 \\
\pm 0.69 \\
c\end{array}$ & $\begin{array}{c}2.80 \\
\pm 0.39 \\
\mathrm{c}\end{array}$ & $\begin{array}{c}\text { (إصابة فقط (إبة } \\
\text { T4 }\end{array}$ \\
\hline$*$ & * & $*$ & * & المعنوية \\
\hline
\end{tabular}

الأحرف الصغيرة المذلفة ضمن كل عمود تثثير الى وجود فرق معنوي :

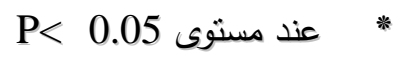

معدلات وزن الجسم الحي والزيادة الوزنية واستهلالك العلف وارتفاع في نسبة الهلاكات للمعاملة

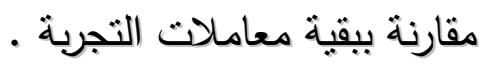
ظهر تحسن في وزن الجسم الحي والزيادة الوزنية واستهلاك العلف لافراخ المعاملات T1 و T2 و نتيجة اضافة المرادف الحيوي الى علائقها لما له من اثر كبير في تحسين اداءها الانتاجي بسبب دور هذه الخميرة وجدارها الخلوي MOS في الحد من الأصابة الجرثومية من خلال الأرتباط 
بهذه الجراثيم ومنعها من اختراق بطانة النسيج الظهاري للأمعاء وطرحها مع الفضلات (20) كما ان

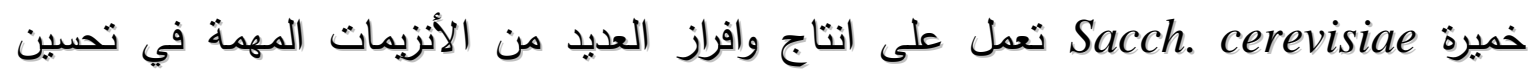

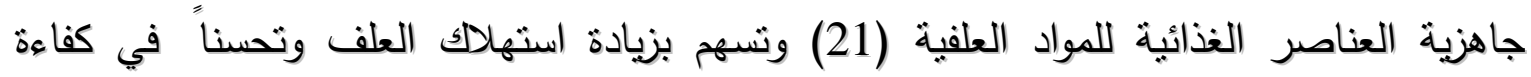
التحويل الغذائي (22) , وكذلك للاور الذي تؤديه خميرة Sacch. cerevisiae الخلطية (23) , وبالنالى خفض نسبة الهاكات.

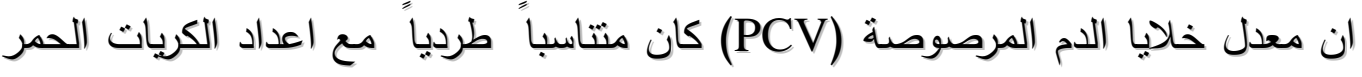

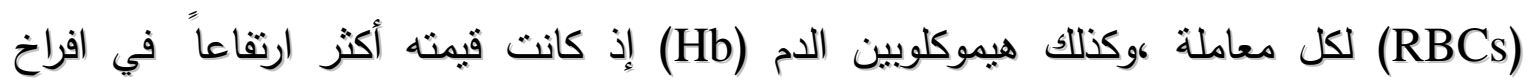

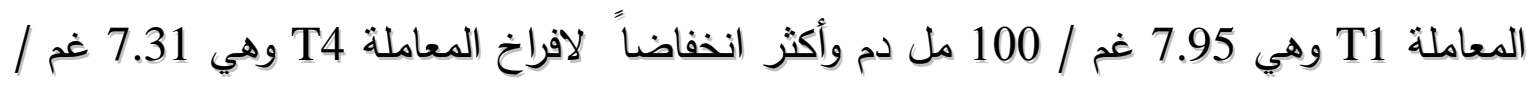

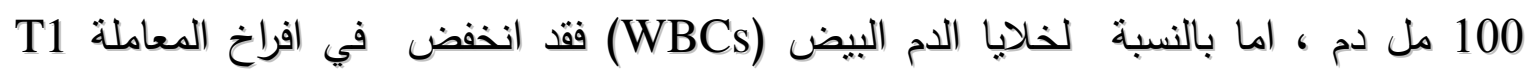

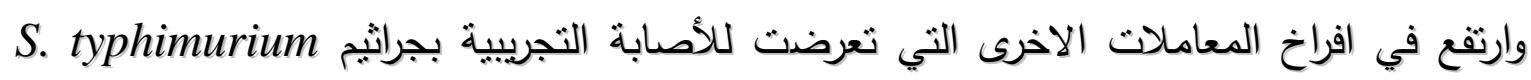

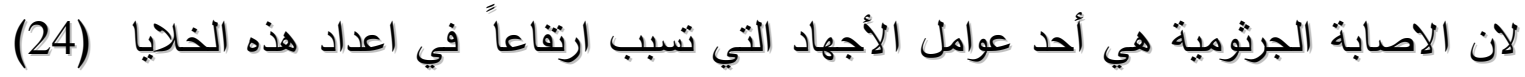

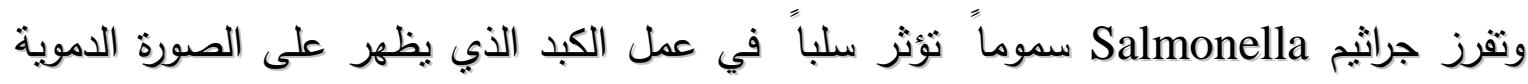
للجسم الحي (19).

\section{المصادر}

1. Jeffery,J.S.(1999).Use of competitive exclusion product for poultry ,P.F.S.:30.

2. Salmenin,S.;A.V.Wright;L.Morelli;P.Mateau;D.Brassart,W.M.Vos.;R.FOnden ; M. Sacelin ; K. Collins ; G. , Mogensen , S. E. Birkeland and T.M.Sandholm.(1998).Demonstration of safety of probiotics a review .Int.J.Food Microbial.,44:93- 106.

3. Schrezenmier,J.Vrese,M.D.(2001).Probiotics , Probiotics and synbiotics approaching adefinition .Am.J.Nutri.vol. 73: 3015-3645.

4. Yeo,J. and K.I.Kim.(1997).Effect of feeding diets containing an antibiotic, Probiotic, Probiotic or Yucca extract on growth and intestinal Urease activity in broiler chicks .Poult.Sci.76:381-385.

5. Craven, S. E. ; N. J. Stern ; S. E. Line ; N. A. Cox, and J. S. Bailey (1997).Reduction of Colstridium perfringes in the ceca of broiler with a mucosal starter culture T.M.or a culture of the Yeast (Supp.7):5124(Abst.).

6. Nisbet, D. J. E. ; Corrier, M. E. Hume, J. A. Byrd , L. H. Stander and R. A. Anderson,(1997).Effcet of $\left(\mathrm{CF}_{3}{ }^{\mathrm{TM}}\right)$.on cecal colonization by Escherichia coli 0157: H7in Broiler chicks .Poultry Sci.76(Suppl.1) 530 Abst.

7. Corrier,D.E.;J.A.Byrd,M.E.Hume.;D.J.Nisbet, and L.Stanker, (1997).Effect of treatment with characterized competitive exclusion culture on a simulkineous 
Salmonella chaling and seeder to contact chick transmission .Poultry Sci.76 (Supp1).:120(Abst.).

8. الثديدي ، شهرزاد محمد (2001). تأثير استخدام نسب من خميرة الخبر والعلف المخمر بها

على الأداء الانتاجي والصفات النوعية لفروج اللحم ، رسالة ماجستير - كلية الزراعة - جامعة الثرة

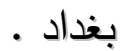

9. العبيدي ، فارس عبد علي وقطان , غادة عبد الخالق وشهرزاد محمد الثنديدي ( 2008 )

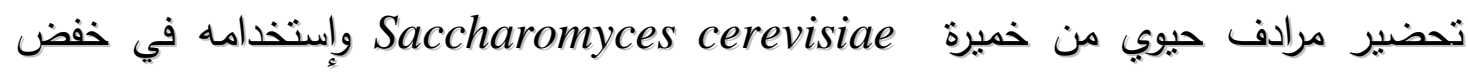

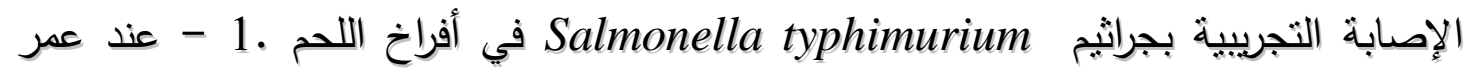

مبكر (1 1 - 15 يوم) .المجلة الطبية البيطرية العراقية.32(2).

10.Pivinick,H.; B.Blanchfield, and D' Aoust,J.Y.(1981).Prevention of Salmonella infection in chicks by pretreatment with faecal cultures from mature chickens (Nurmi cultures)J.Food Protect .44(12):909-916.

11.Miles,A.A.; S.S.Misra, and J.O.Irwin, (1938).The estimation of the bactericidal power of blood J.Hyg.Camp.38:739-746.

12.Line,J.E.;J.S.;Baily,N.A.Cox, and N.J. Steven,(1997).Yeast treatment to reduce Salmonella and Campylobacter population associated with broiler chickens subjected to transport stress.Poultry Sci.76:1227- 1231.

13.Varley,H.A.H.GrawenlockandM.Bell.(1980).PracticalClinical Biochem istry 5thed William Heinemann Medical Books.Ltd.,London.

14.Natt M.P. and C.A. Herrick(1952).A new blood diluent for counting the erythrocytes and Leukocytes of the chicken.Poultry Sci.31:735- 738.

15.Archer,P.K.(1965).Haematological techniques for use in animals Oxford Black Scientific Publications.

16.SAS,Institue,(2001).SA/TAT user's Guide version G. 4th ed SAS Institue Gary,NC.

17.Zhang-Barber, L . ; Turner,A.K. and Barrow , P . A . ( 1999 ). Vaccination for contol of Salmonella in Poultry.Vaccine.17:2538.2545.

18.Muir,W.I.; W.L. Bryden, and A.J. Hudband , (2000).Immunity Vaccination and $t$ he avian intestinal tract . Dvelop . Comparat . Immune.24:325-342.

19.Williams,J.E.(1984). Paralytic infections,In: Hofstand, M.S., Barnes, H.J.;Calnek,B.W.;Reid,W.M. and Yoder,Jr.,H.W.Eds.) Diseases of poultry .pp:91-129.Iowa state uni.press.Ames,Iowa.USA.

20.CFNP Center for Food and Nutrition Policy(CFNP) Viriginia Tech-Alexandria August 2002.

21.Day,E.J.B.C.DiLworth, and S.Omer.(1987).Effect of varying levels of phosphorous and live yeast culture in caged laying diets.Poultry Sci.66:1402141. 
22.Gurrero,R. and G.Hoyos.(1991).Direct fed microbials and cidifiers for poultry .P.407-411. alltechs 7th Ann.Sym. On .Biotechnology in the feed industry .In: T.P. loyns and K. A. Jacques .Eds ,Lough Bovough ,Leciershire, USA.

23.Verword,D.J.;A.J.Oliver,M.M.Herton and Vander walt (1998). Main training health and performance in the young ostrich : Applications for a Mannan Oligosaccharide Pp:539-551. Proc.Altech's 14th Ann.Sym.On Biotechnology in the feed industry inTp:Lyons and K.A. acques, eds.Longborough, Leicestershir, U.S.A.

24.Sturkie,P.D(1986).Avian Physiology 4th ed Springer-Verlag.New York. 